\title{
Geant4 Standard and Low Energy electromagnetic libraries
}

\author{
Vladimir Ivanchenko ${ }^{1,2, a}$ and Sebastien Incerti ${ }^{3}$ on behalf of the Geant4 Collaboration \\ ${ }^{1}$ CERN, CH1211Geneva 23, Switzerland \\ ${ }^{2}$ Geant4 Associates International Ltd, United Kingdom \\ ${ }^{3}$ Université of Bordeaux 1, CNRS/IN2P3, CENBG, 33175 Gradignan, France
}

\begin{abstract}
Geant4 is a Monte Carlo toolkit well established for large scale simulations for high energy physics, medicine, space science and other applications. Electromagnetic (EM) physics libraries are key components of these simulations. In this work we describe the Standard and Low Energy EM libraries available in Geant4 version 10.3 including descriptions of new models and interfaces relevant for dark matter searches and limits of applicability for these models.
\end{abstract}

\section{Introduction}

The Geant4 Monte Carlo toolkit [1-3] is used for simulation of many particle physics experiments including ATLAS and CMS. It is a natural choice to use Geant4 for the design of new experiments to search for dark matter particles. Light dark matter particle may have a coupling with leptons and gammas. A possible dark matter signal in this case should be seen in processes of production of electron, positron, gamma, or in missing energy experiments. Such a fix target experiment - NA64 already started at CERN SPS [4].

In Geant4, such processes are simulated with electromagnetic (EM) physics models, which are organised in standard and low-energy sub-libraries. In this work, a status of Geant4 EM sub-libraries will be described with focus on aspects important for dark matter search. We will discuss the recent Geant4 version 10.3 (December, 2016).

\section{GEANT4 electromagnetic physics}

Geant4 EM processes and models are implemented using a common interface for all sub-libraries [3], [5], [6]. This allows use of a combination of low-energy and highenergy models in a transparent way. In user applications, it is possible additionally define specific models for particle types and detector regions. For sampling of final states, it is also possible to exchange angular generators between standard and low-energy models.

In this work, we will discuss EM physics models which are important for search of the dark matter particles having EM coupling: conversion to e+e- pair, bremsstrahlung, and positron annihilation.

\subsection{Gamma models}

The full list of gamma models and their applicability area are discussed in our recent review [3]. The most important process for dark matter search is the gamma conversion process in atomic field. Models from the standard and low-energy EM sub-libraries in recent Geant4 versions have practically the same conversion cross section (Fig.1). They are based on the first Born approximation. For high energies $(\mathrm{E}>80 \mathrm{GeV})$ a new relativistic model was developed which takes into account LPM suppression [7].

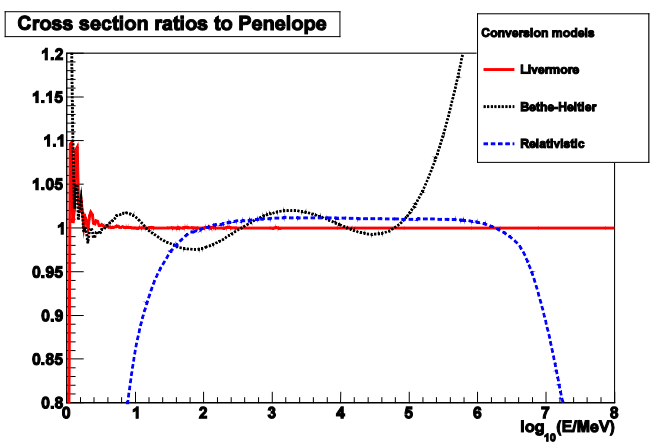

Figure 1. Ratios of cross section of gamma conversion in tungsten for various Geant4 models to the Penelope cross section. The relativistic model does not take into account atomic effects at threshold, but is the only model implemented the LPM suppression at high energy. Some difference at reaction threshold between Livermore and Penelope is due to different binning of cross section data.

\section{a Corresponding author: Vladimir.Ivantchenko@cern.ch}


In the Bethe-Heilter model, the Geant3 parameterisation of the cross section is implemented (Fig.1). The relative accuracy of this parameterisation is estimated as $2 \%$ for all atoms, and the upper limit of applicability is clearly seen in the plot. The relativistic model uses analytical expressions for cross sections. Penelope and Livermore models are more accurate at the reaction threshold and for high energy they use the same theoretical formulas as the relativistic one but without LPM suppression.

All these cross sections agree within $2 \%$ in the energy range $100 \mathrm{MeV}-100 \mathrm{GeV}$ for all atoms. These models do not include high order corrections, so their absolute accuracy may be estimated on the level of a few percent. Sampling of final state in the Livermore model is different from others, because it includes a possibility of combining two models: one for sampling of the reaction with a nucleus, and another for reaction with atomic electrons. In the last case, atomic electron recoil is produced as a secondary particle [3], [8], which is a possible background for the dark matter signal search in fix target experiments.

\subsection{Energy loss models}

For electron and positron the main energy loss processes are ionisation and bremsstrahlung [3], [5-7]. In the case of ionisation, one secondary delta-electron is produced. The process of bremsstrahlung has two components: interaction with atomic nucleus or with atomic electrons. In current EM models electron recoil is not yet simulated but this is foreseen in the future. For Geant4 10.3, a new process was implemented of e+e- pair production by electron or positron. For that, the approach developed for the simulation for the e+e- pair production by muon is used [9]. In Fig.2 two partial cross sections for energy transfer above $10 \mathrm{MeV}$ are shown. The process of e+epair production has much less cross section compared with bremsstrahlung but may contribute to background to dark matter signal if an energy transfer is large enough. In Geant4 10.3, this new process is included into Opt3 and Opt4 physics lists.

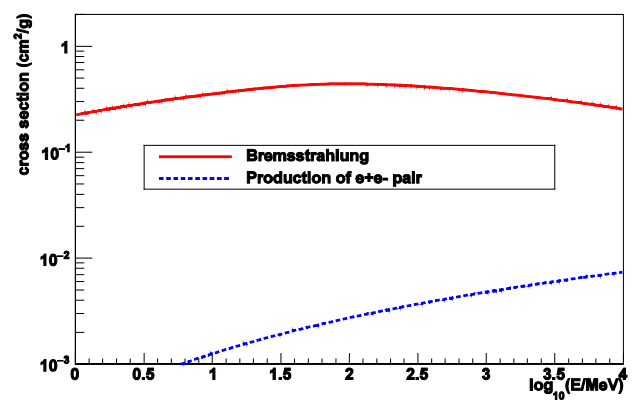

Figure 2. Partial cross section of bremsstrahlung (solid line) and e+e- pair production (dotted line) in lead by electrons with energy transfer above $10 \mathrm{MeV}$ as a function of the projectile electron energy.

\subsection{Positron annihilation}

Two gamma annihilation of a positron is the main positron annihilation Geant4 process. For version 10.3, a correlation of linear polarisation of produced photons was implemented [10]. Additionally a new UI command and a $\mathrm{C}++$ interface were added to enable rare high energy processes of positron annihilation to muons and hadrons [9], [11]. These UI commands are available for all reference physics lists.

\section{Configuration of EM physics}

We recommend [3] using reference physics lists or building custom physics list using physics constructors from the Geant4 distribution. These approaches guarantees usage of a well validated combination of physics models. However, a set of reference physics lists cannot cover all use cases. In particular, for simulation of direct interaction of dark matter particles with media, additional models should be added into application. For that, a model may be easily prepared using inheritance from the existing models for gamma, electron, or positron interactions. For estimation of background for dark matter search, some of the necessary EM models are already available with Geant4 10.3 but not all. A user can add a new custom model on top of any physics list using existing interfaces to EM physics.

\section{Summary}

The existing set of EM physics sub-libraries of Geant4 provides a possibility for designing experiments for the search of the dark matter particles having coupling with gamma, electrons, and positrons. Additional development for the next leading order corrections to EM physics models is required within Geant4 but also may be implemented at the level of user applications.

\section{References}

1. S. Agostinelli, et al, Nucl. Instrum . Methods Phys. Res. A. 506, 250 (2003)

2. J. Allison, et al., IEEE Trans. Nucl. Sci. 53, 270 (2006)

3. J. Allison, et al., Nucl. Instrum. Methods Phys. Res. A 835, 186 (2016)

4. NA64 Collaboration, arXiV:1610.02988 [hep-ph]

5. J. Apostolakis, et al., Radiat. Phys. Chem., 78, 859 (2009)

6. V. Ivanchenko, et al., Prog. Nucl., Sci., Technol. 2, 898 (2011)

7. V. N. Ivanchenko, et al., J. Phys : Conf. Ser. 513, 022015 (2014)

8. M. L. Iparraquirre, G. O. Depaola, Eur. Phys. J. C 71, 1778 (2011)

9. A. G. Bogdanov, et al., IEEE Trans. Nucl. Sci. 53, 513 (2006).

10. M. H. L. Pryce, J. C. Ward, Nature 4065, 435 (1947)

11. J. Apostolakis, et al., J. Phys : Conf. Ser. 664, 072021 (2015) 